\title{
Neutrino Electromagnetic Form Factor and Oscillation Effects on Neutrino Interaction With Dense Matter
}

\author{
C. K. Williams, P. T. P. Hutauruk, A. Sulaksono, T. Mart \\ Departemen Fisika, FMIPA, Universitas Indonesia, Depok 16424, Indonesia
}

(Dated: August 4, 2021)

\begin{abstract}
The mean free path of neutrino - free electron gas interaction has been calculated by taking into account the neutrino electromagnetic form factors and the possibility of neutrino oscillation. It is shown that the form factor effect becomes significant for a neutrino magnetic moment $\mu_{\nu} \geq 10^{-10} \mu_{B}$ and for a neutrino radius $R \geq 10^{-6} \mathrm{MeV}^{-1}$. The mean free path is found to be sensitive to the $\nu_{e}-\nu_{\mu}$ and $\nu_{e}-\nu_{e}^{R}$ transition probabilities.

PACS numbers: 13.15.+g, 13.40.Gp, 25.30.Pt, 97.60.Jd, 14.60.Pq
\end{abstract}

Neutrino interaction with dense matter plays an important role in astrophysics, e.g., in the formation of supernova and the cooling of young neutron stars 1, 2, 3, 4, 5, 6, 7, 8]. Earlier calculation on neutrino interactions with electrons gas, dense and hot matter, based on the standard model has been performed by Horowitz and Wehrberger 2, 3]. Some relativistic calculations of neutrino mean free path in hot and dense matter have been also done in Refs. [4, 5, 6, 7]. Recently, due to a demand on a more realistic neutrino mean free path for supernova simulations, a mean free path calculation by taking into account the weak magnetism of nucleons has been also performed [8].

However, certain phenomena such as solar neutrinos, atmospheric neutrinos problems, and some astrophysics and cosmology arguments need explanations beyond the standard model assumption of neutrino's properties such as neutrino oscillation 9, 10], the helicity flipping of neutrinos 11, 12, 13, 14] and neutrino electromagnetic form factors. We note that the upper bound of the neutrino magnetic moment extracted from the SuperKamiokande solar data [15, 16] falls in the range of $(1.1-1.5) \times 10^{-10} \mu_{B}$, where $\mu_{B}=e / 2 m_{e}$ stands for the Bohr magneton. Other experimental limits [17, 18] give $\mu_{\nu}<1.0 \times 10^{-10} \mu_{B}$, whereas signals from Supernova 1987A (SN1987A) require that $\mu_{\nu} \leq 1.0 \times 10^{-12} \mu_{B}$. These bounds have been derived by considering the helicity flipping neutrino scattering in a supernova core [19]. In the case of random magnetic fields inside the sun, one can obtain a direct constraint on the neutrino magnetic moment of $\mu_{\nu} \leq 1.0 \times 10^{-12} \mu_{B}$, similar to the bounds obtained from the star cooling [20]. In addition, data from muon neutrino- and anti neutrino-electron scatterings [21, 22] and a close examination to the data over the years from Kamiokande II and Homestake according to Mourão et al. [24], similarly give a neutrino average squared radius $R^{2} \sim 25 \times 10^{-12} \mathrm{MeV}^{-2}$ with $R^{2}=\left\langle R_{V}^{2}\right\rangle$ $+\left\langle R_{A}^{2}\right\rangle$. The definitions of $\left\langle R_{V}^{2}\right\rangle$ and $\left\langle R_{A}^{2}\right\rangle$ will be explained later.

Therefore, in connection with the demand on realistic neutrino mean free path in dense and hot matter, an extension of the previous study [4, 5, 6, 7, 8] which takes into account the electromagnetic form factors of neutri- nos and neutrino oscillations is inevitable. As a first step before that, in this report we calculate the mean free path of neutrino-free electrons gas where those effects are included. Here we assume that neutrinos are massless and the RPA correlations can be neglected. Furthermore, we use zero temperature approximation in this calculation.

In the standard model, where the momentum transfer is much less than the $W$ mass, direct $Z^{0}$ and $W^{ \pm}$contributions to the matrix element $\mathcal{M}$ can be written as an effective four-point coupling $[\underline{3}, 22]$

$$
\mathcal{M}_{W}=\frac{G_{F}}{\sqrt{2}}\left[\bar{U}\left(k^{\prime}\right) \gamma^{\mu}\left(1+\gamma^{5}\right) U(k)\right]\left[\bar{U}\left(p^{\prime}\right) J_{\mu} U(p)\right],
$$

where $G_{F}$ is the coupling constant of weak interaction, $U(k)$ and $U(p)$ are neutrino and electron spinors, respectively, and the current $J_{\mu}$ is defined by

$$
J_{\mu}=\gamma_{\mu}\left(C_{V}+C_{A} \gamma^{5}\right) .
$$

The vector and axial vector couplings $C_{V}$ and $C_{A}$ can be written in terms of Weinberg angle $\theta_{W}$ (where $\sin ^{2} \theta_{W} \approx$ 0.223 [3, [4]) as $C_{V}=2 \sin ^{2} \theta_{W} \pm 1 / 2$ and $C_{A}= \pm 1 / 2$ (the upper sign is for $\nu_{e}$, the lower sign is for $\nu_{\mu}$ and $\nu_{\tau}$ ).

The electromagnetic properties of Dirac neutrinos are described in terms of four form factors, i.e., $f_{1 \nu}, g_{1 \nu}, f_{2 \nu}$ and $g_{2 \nu}$, which stand for the Dirac, anapole, magnetic, and electric form factors, respectively. The matrix element for the neutrino-electron interaction which contains electromagnetic form factors reads 22]

$$
\begin{aligned}
\mathcal{M}_{E M} & =\frac{4 \pi \alpha}{q^{2}}\left[\bar{U}\left(p^{\prime}\right) \gamma_{\mu} U(p)\right]\left\{\overline { U } ( k ^ { \prime } ) \left[f_{m \nu} \gamma^{\mu}\right.\right. \\
& \left.\left.+g_{1 \nu} \gamma^{\mu} \gamma^{5}-\left(f_{2 \nu}+i g_{2 \nu} \gamma^{5}\right) \frac{P^{\mu}}{2 m_{e}}\right] U(k)\right\} .
\end{aligned}
$$

where $f_{m \nu}=f_{1 \nu}+\left(m_{\nu} / m_{e}\right) f_{2 \nu}, P^{\mu}=k^{\mu}+k^{\mu \prime}, m_{\nu}$ and $m_{e}$ are neutrino and electron masses, respectively. In the static limit, the reduced Dirac form factor $f_{1 \nu}$ and the neutrino anapole form factor $g_{1 \nu}$ are related to the vector and axial vector charge radii $\left\langle R_{V}^{2}\right\rangle$ and $\left\langle R_{A}^{2}\right\rangle$ through 22]

$$
f_{1 \nu}\left(q^{2}\right)=\frac{1}{6}\left\langle R_{V}^{2}\right\rangle q^{2} \quad \text { and } \quad g_{1 \nu}\left(q^{2}\right)=\frac{1}{6}\left\langle R_{A}^{2}\right\rangle q^{2} .
$$


In the limit of $q^{2} \rightarrow 0, f_{2 \nu}$ and $g_{2 \nu}$ define respectively the neutrino magnetic moment $\mu_{\nu}^{m}=f_{2 \nu}(0) \mu_{B}$ and the (CP violating) electric dipole moment $\mu_{\nu}^{e}=g_{2 \nu}(0) \mu_{B}$ [22, 23]. Here we use $\mu_{\nu}^{2}=\mu_{\nu}^{m}{ }^{2}+\mu_{\nu}^{e}{ }^{2}$.

Next, we can obtain the differential cross section per volume $V$ for scattering of neutrinos with the initial energy $E_{\nu}$ and final energy $E_{\nu}^{\prime}$ on the electrons gas. It consists of the contributions from weak (W) interaction, electromagnetic (EM) interaction, as well as their interference (INT) term, i.e.,

$$
\left(\frac{1}{V} \frac{d^{3} \sigma}{d^{2} \Omega^{\prime} d E_{\nu}^{\prime}}\right)_{\nu_{e}}=-\frac{1}{16 \pi^{2}} \frac{E_{\nu}^{\prime}}{E_{\nu}}\left[\left(\frac{G_{F}}{\sqrt{2}}\right)^{2} L_{\nu}^{\mu \nu} \Pi_{\mu \nu}^{\operatorname{Im}(\mathrm{W})}+\left(\frac{4 \pi \alpha}{q^{2}}\right)^{2} L_{\nu}^{\mu \nu} \Pi_{\mu \nu}^{\operatorname{Im}(\mathrm{EM})}+\frac{8 G_{F} \pi \alpha}{q^{2} \sqrt{2}} L_{\nu}^{\mu \nu} \Pi_{\mu \nu}^{\operatorname{Im}(\mathrm{INT})}\right] .
$$

For each contribution, the neutrino tensors are given by

$$
\begin{gathered}
L_{\nu}^{\mu \nu(\mathrm{W})}=8\left[2 k^{\mu} k^{\nu}-\left(k^{\mu} q^{\nu}+k^{\nu} q^{\mu}\right)+g^{\mu \nu}(k \cdot q)-i \epsilon^{\alpha \mu \beta \nu} k_{\alpha} k_{\beta}^{\prime}\right], \\
L_{\nu}^{\mu \nu(\mathrm{EM})=}=4\left(f_{m \nu}^{2}+g_{1 \nu}^{2}\right)\left[2 k^{\mu} k^{\nu}-\left(k^{\mu} q^{\nu}+k^{\nu} q^{\mu}\right)+g^{\mu \nu}(k \cdot q)\right]-8 i f_{m \nu} g_{1 \nu} \epsilon^{\alpha \mu \beta \nu}\left(k_{\alpha} k_{\beta}^{\prime}\right) \\
-\frac{f_{2 \nu}^{2}+g_{2 \nu}^{2}}{m_{e}^{2}}(k \cdot q)\left[4 k^{\mu} k^{\nu}-2\left(k^{\mu} q^{\nu}+q^{\mu} k^{\nu}\right)+q^{\mu} q^{\nu}\right], \\
L_{\nu}^{\mu \nu(\mathrm{INT})}=4\left(f_{m \nu}+g_{1 \nu}\right)\left[2 k^{\mu} k^{\nu}-\left(k^{\mu} q^{\nu}+k^{\nu} q^{\mu}\right)+g^{\mu \nu}(k \cdot q)-i \epsilon^{\alpha \mu \beta \nu} k_{\alpha} k_{\beta}^{\prime}\right],
\end{gathered}
$$

whereas the polarizations read

$$
\begin{aligned}
\Pi_{\mu \nu}^{\operatorname{Im}(\mathrm{W})} & =C_{V}^{2} \Pi_{\mu \nu}^{\operatorname{Im} V}+2 C_{V} C_{A} \Pi_{\mu \nu}^{\operatorname{Im}(V-A)}+C_{A}^{2} \Pi_{\mu \nu}^{\operatorname{Im} A}, \\
\Pi_{\mu \nu}^{\operatorname{Im}(\mathrm{EM})} & =\Pi_{\mu \nu}^{\operatorname{Im} V}, \\
\Pi_{\mu \nu}^{\operatorname{Im}(\mathrm{INT})} & =C_{V} \Pi_{\mu \nu}^{\operatorname{Im} V}+C_{A} \Pi_{\mu \nu}^{\operatorname{Im}(V-A)} .
\end{aligned}
$$

Due to the current conservation and translational invariance, the vector polarization $\Pi_{\mu \nu}^{\operatorname{Im} V}$ consists of two independent components which we choose to be in the frame of $q^{\mu} \equiv\left(q_{0},|\vec{q}|, 0,0\right)$, i.e.,

$$
\begin{aligned}
& \Pi_{T}^{\operatorname{ImV}}=\Pi_{22}^{V}=\Pi_{33}^{V} \quad \text { and } \\
& \Pi_{L}^{\operatorname{ImV}}=-\left(q_{\mu}^{2} /|\vec{q}|^{2}\right) \Pi_{00}^{V} .
\end{aligned}
$$

The axial-vector and the mixed pieces are found to be

$$
\Pi_{\mu \nu}^{\operatorname{Im}(\mathrm{V}-\mathrm{A})}(q)=i \epsilon_{\alpha \mu 0 \nu} q_{\alpha} \Pi_{V A},
$$

and

$$
\Pi_{\mu \nu}^{\operatorname{Im} A}(q)=\Pi_{\mu \nu}^{\operatorname{Im} V}(q)+g_{\mu \nu} \Pi_{A} .
$$

The explicit forms of $\Pi_{22}^{V}, \Pi_{00}^{V}, \Pi_{V A}$ and $\Pi_{A}$ are given in Ref. [3]. Thus the analytical form of Eq. (5) can be obtained from the contraction of every polarization and neutrino tensors couple $\left(L^{\mu \nu} \Pi_{\mu \nu}\right)$.
If we take into account the possibility of the $\nu_{e}-\nu_{\mu}$ transition, the cross section can be written in the form of [25, 26]

$$
\frac{d^{3} \sigma}{d^{2} \Omega^{\prime} d E^{\prime}}=P_{e e}\left(\frac{d^{3} \sigma}{d^{2} \Omega^{\prime} d E^{\prime}}\right)_{\nu_{e}}+\left(1-P_{e e}\right)\left(\frac{d^{3} \sigma}{d^{2} \Omega^{\prime} d E^{\prime}}\right)_{\nu_{\mu}}
$$

Here $\left(d^{3} \sigma / d^{2} \Omega^{\prime} d E^{\prime}\right)_{\nu_{e}}$ is the cross section of the $\nu_{e}-e$ scattering. If $C_{V}$ and $C_{A}$ are replaced with $C_{V}-1$ and $C_{A}-1$, respectively, then the cross section becomes $\left(d^{3} \sigma / d^{2} \Omega^{\prime} d E^{\prime}\right)_{\nu_{\mu}}$, i.e., the cross section of the $\nu_{\mu}-e$ scattering. $P_{e e}$ is the $\nu_{e}$ 's flavor survival probability as a function of the neutrino energy.

Due to the assumption of massless neutrino, the $\nu_{e}$ helicity flip from left- to right-handed is only possible through it's dipole moment. Thus, the cross section after taking into account this possibility $\left(\nu_{e}-\nu_{e}^{R}\right.$ transition) reads [17]

$$
\frac{d^{3} \sigma}{d^{2} \Omega^{\prime} d E^{\prime}}=\left(1-P_{L L}\right)\left(\frac{d^{3} \sigma}{d^{2} \Omega^{\prime} d E^{\prime}}\right)_{L R}+P_{L L}\left(\frac{d^{3} \sigma}{d^{2} \Omega^{\prime} d E^{\prime}}\right)_{\nu_{e}} .
$$

where $\left(d^{3} \sigma / d^{2} \Omega^{\prime} d E^{\prime}\right)_{L R}$ is the $\nu_{e}-e$ scattering via neutrino dipole moment and $P_{L L}$ is the probability of $\nu_{e}$ to 

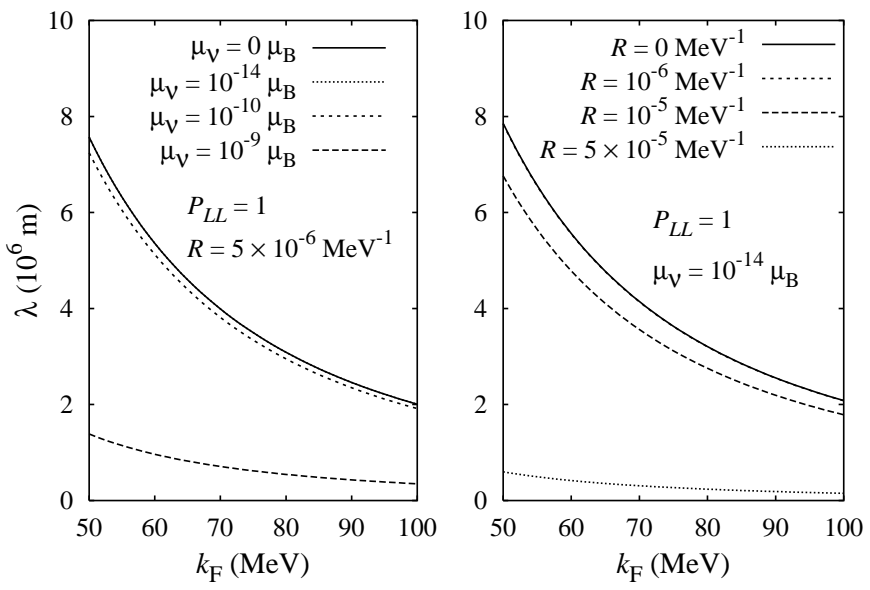

FIG. 1: Total mean free path compared to the mean free path of weak interaction with various neutrino magnetic moments $\mu_{\nu}$ and radii $R$ as a function of Fermi momentum $k_{F}$. In the left panel the neutrino charge radius is fixed, while the neutrino magnetic moment is varied. In the right panel, we fix the neutrino magnetic moment, but vary the neutrino radius.
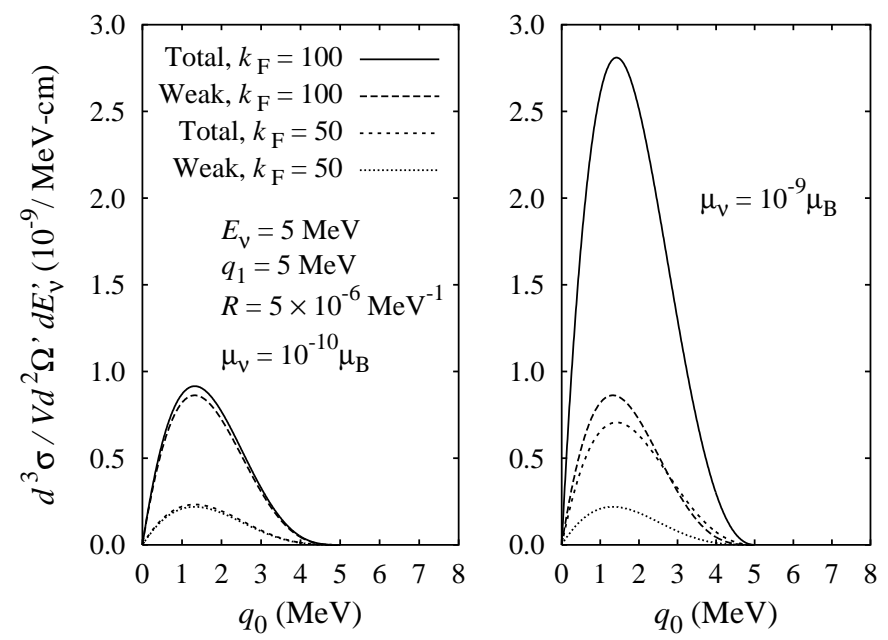

FIG. 2: Total cross section compared to the cross section of weak interaction as a function of energy transfer $q_{0}$ where momentum transfer $q_{1}$ is fixed. Here, two different neutrino magnetic moments $\mu_{\nu}$ and Fermi momenta with a same neutrino charge radius are used. In the left panel we use $\mu_{\nu}=10^{-10} \mu_{B}$, while in the right panel $\mu_{\nu}=10^{-9} \mu_{B}$.

be still left handed.

Finally we can compute the mean free path from Eqs. (5), (12), and (13), by using

$$
\frac{1}{\lambda\left(E_{\nu}\right)}=\int_{q_{0}}^{2 E_{\nu}-q_{0}} d|\vec{q}| \int_{0}^{2 E_{\nu}} d q_{0} \frac{|\vec{q}|}{E_{\nu}^{\prime} E_{\nu}} 2 \pi \frac{1}{V} \frac{d^{3} \sigma}{d^{2} \Omega^{\prime} d E_{\nu}^{\prime}} .
$$

In this calculation we use a neutrino energy of $5 \mathrm{MeV}$.

Figure 1 shows the total mean free path compared to the mean free path of weak interaction with various neutrino effective moments $\mu_{\nu}$, and neutrino charge radii $R$.
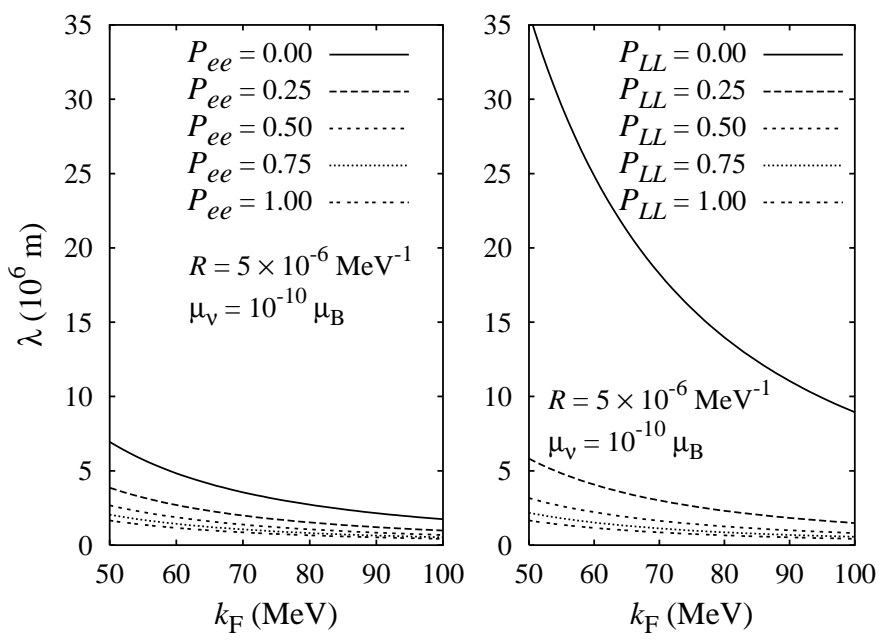

FIG. 3: Total mean free path of $\nu_{e}$ that allows for $\nu_{e}-\nu_{\mu}$ and $\nu_{e}-\nu_{e}^{R}$ transitions with various $P_{L L}$ and $P_{e e}$ as a function of Fermi momentum $k_{F}$. In the left panel we vary the $\nu_{e}$ 's flavor survival probability, while in the right panel the helicity flipping probability of neutrino is varied.

The total mean free path is the coherent sum of the weak, electromagnetic and the interference contributions.

There are also evidences that $R^{2} \approx 10^{-32} \mathrm{~cm}^{-2}$ or $R^{2} \approx 25 \times 10^{-12} \mathrm{MeV}^{-2}[21,22,24]$. Therefore, in the left panel of Fig. 10 use $R=5 \times 10^{-6} \mathrm{MeV}^{-1}$ and vary $\mu_{\nu}$ between 0 and $10^{-9} \mu_{B}$. In the right panel, we use $\mu_{\nu}=$ $10^{-12} \mu_{B}$ as the strongest bound on the neutrino magnetic moment while $R$ is varied between 0 and $5 \times 10^{-5} \mathrm{MeV}^{-1}$.

It is evident from the left panel of Fig. 1 that for fixed $R$, the mean free path increases rapidly only after $\mu_{\nu}=10^{-10} \mu_{B}$. As we can see from Fig. 22 this increment is due to the significant difference between total and weak cross sections starting from $\mu_{\nu}=10^{-10} \mu_{B}$. The summation of the longitudinal and transversal terms of the electromagnetic contribution is responsible for this. The right panel shows that for fixed $\mu_{\nu}$, the total mean free path and the mean free path of weak interaction show significant variance for $R \geq 10^{-6} \mathrm{MeV}^{-1}$. This is also due to the fact that the summation of the longitudinal and transversal terms of the electromagnetic part of the cross section increases rapidly starting at $R=10^{-6} \mathrm{MeV}^{-1}$.

Figure 3 shows the effects of neutrino oscillations on the neutrino mean free path. In this case we do not calculate the transition probabilities. Instead, we only study the variation of neutrino mean free path with respect to the transition probabilities of a left handed massless neutrino electron, $\nu_{e}$, oscillates to a left handed massless neutrino muon, $\nu_{\mu}$, or flips to a right handed neutrino electron, $\nu_{e}^{R}$.

By comparing the possibility of $\nu_{e}-\nu_{\mu}$ transition (left panel of Fig. (3) and $\nu_{e}-\nu_{e}^{R}$ transition (right panel), we can clearly see that these effects lengthen the neutrino mean free path, where the rate depends on their survival probabilities. For smaller $P_{L L}$ (large flipping possibility), 
the path increment becomes more significant. This effect can be traced back to the value of $\left(d^{3} \sigma / d^{2} \Omega^{\prime} d E^{\prime}\right)_{L R}$ in Eq. (13) which is smaller than that of $\left(d^{3} \sigma / d^{2} \Omega^{\prime} d E^{\prime}\right)_{\nu_{e}}$. On the other hand, for small $P_{e e}$ the possibility of $\nu_{e}-\nu_{\mu}$ oscillation does not change the neutrino mean free path dramatically. This fact arises because the difference between $\left(d^{3} \sigma / d^{2} \Omega^{\prime} d E^{\prime}\right)_{\nu_{\mu}}$ and $\left(d^{3} \sigma / d^{2} \Omega^{\prime} d E^{\prime}\right)_{\nu_{e}}$ in Eq. (12) is not as large as in the case of Eq. (13). Therefore different from the mean free path with flavor changing possibility, the mean free path with helicity flipping possibility depends strongly on the value of $\mu_{\nu}$. For example we have also found that with decreasing $P_{L L}$ the mean free path grows more rapidly when we use $\mu_{\nu}=10^{-12} \mu_{B}$ rather than $\mu_{\nu}=10^{-10} \mu_{B}$.

In conclusion, we have studied the sensitivity of the neutrino mean free path to the neutrino electromagnetic form factors and neutrino oscillations. It is found that the electromagnetic form factor has a significant role if $\mu_{\nu} \geq 10^{-10} \mu_{B}$ and $R \geq 10^{-6} \mathrm{MeV}^{-1}$. We note that these values are larger than their largest upper bounds. It would be interesting to see whether or not such phenomenon would also appear if contributions from the neutrino-nucleon scatterings were taken into account. Future calculation should address this question. The mean free path is also found to be sensitive to the neutrino oscillations and depends on the transition probabilities of $\nu_{e}-\nu_{\mu}$ and $\nu_{e}-\nu_{e}^{R}$. This result clearly indicates that realistic mean free path calculations in the future should be performed with appropriate values of the $\nu_{e}-\nu_{\mu}$ and $\nu_{e}-\nu_{e}^{R}$ transition probabilities.

TM and AS acknowledge the support from the QUE project.
[1] Sanjay Reddy, M. Prakash, J. M. Lattimer, J. A. Pons, Phys. Rev. C 59, 2888 (1999).

[2] C. J. Horowitz and K. Wehrberger, Phys. Rev. Lett. 66, 272 (1991);

[3] C. J. Horowitz and K. Wehrberger, Nucl. Phys. A531, 665 (1991); Phys. Lett. B 226, 236 (1991).

[4] R. Niembro, P. Bernados, M. López-Quele, S. Marcos, Phys. Rev. C 64, 055802 (2001).

[5] L. Mornas and A. Parez, Eur. Phys. J. A 13, 383 (2002).

[6] L. B. Leinson, Nucl. Phys. A 707, 543 (2002).

[7] G. Fabbri and F. Matera, Phys. Rev. C 54, 2031 (1996).

[8] C. J. Horowitz and M. A. Pérez-García, Phys. Rev. C 68, 025803 (2003).

[9] T. K. Kuo and J. Pantaleone, Rev. Mod. Phys. 61, 937 (1992); and references therein.

[10] J. Pulido, Phys. Rept. 211, 167 (1992); and references therein.

[11] A. Ayala, J. C. D'Olivo and M. Tores, Phys. Rev. D 59, 111901(1999).

[12] K. Enqvist, P. Keraenen and J. Maalampi, Phys. Lett. B 438, 295 (1998).

[13] K. J. F. Gaemers, R. Gandhi, J. M. Lattimer, Phys. Rev. D 40, 309 (1989).
[14] W. Grimus, P. Stockinger, Phys. Rev. D 57, 1762 (1998).

[15] D. W. Liu et al., Phys. Rev. Lett 93, 021802 (2004).

[16] J. F. Beacom and P. Vogel, Phys. Rev. Lett 83, 5222 (1999).

[17] W. Grimus et al., Nucl. Phys. B 648, 376 (2003).

[18] Z. Daraktchieva et al., Phys. Lett B 564, 190 (2003).

[19] N. Nunokawa, R. Tomás and J. W. F. Valle, Astropart. Phys. 11, 317 (1999); and references therein.

[20] O. G. Miranda, T. I. Rashba, A. I. Rez and J. W. F. Valle, Phys. Rev. Lett 93, 051304 (2004).

[21] R. C. Allen et al., Phys. Rev. D 43, 1 (1991).

[22] B. K. Kerimov, M. Ya Safin and H. Nazih, Izv. Ross. Akad. Nauk. SSSR. Fiz. 52, 136 (1998).

[23] Enrico Nardi, AIP Conf. Proc. 670, 118 (2003).

[24] A. M. Mourão, J. Pulido, and J. P. Ralston, Phys. Lett. B 285, 364 (1992).

[25] H. P. Simanjuntak and A. Sulaksono, Mod. Phys. Lett. 9A, 2179 (1994); A. Sulaksono and H. P. Simanjuntak, Solar Phys. 151, 205 (1994).

[26] J. Morgan, Phys. Lett. B102, 247 (1981); M. Fukugita and S. Yazaki, Phys. Rev. D 36, 3817 (1987). 